\title{
NEUROMUSCULAR DISORDERS AMENABLE TO WHEAT GERM OIL THERAPY
}

\author{
BY \\ REUBEN RABINOVITCH, WILLIAM C. GIBSON, \\ and DONALD MCEACHERN
}

\section{From the Department of Neurology and Neurosurgery, McGill University, and the Montreal Neurological Institute, Montreal}

There has been a natural desire to find some neuromuscular disorder in man equivalent to the nutritional muscular dystrophy which occurs in animals deprived of wheat germ factors or of vitamin E. So far the search has been unsuccessful. Our own therapeutic trials of wheat germ oil were begun in 1936, and since that time 107 patients, most of them with neuromuscular disorders, have been studied. In each case treatment has been carried out over many months, and in some cases for many years.

In certain conditions, for example, progressive motor neuron disease, no favourable effect has been noted. In 12 patients with other disorders improvement or recovery has been sufficiently dramatic to make us believe that treatment was responsible. These conditions include dermatomyositis, menopausal muscular dystrophy, and some cases of progressive muscular dystrophy.

\section{Review of the Literature}

Evans and Bishop (1922) found that female rats deprived of certain foodstuffs were unable to carry on normal gestation. The factor necessary to prevent abortion was found plentifully in green vegetables, cereals and seeds, and especially in wheat germ oil. It was tentatively named vitamin $X$ and later vitamin E. It has become popularly known as the "anti-sterility vitamin", although the latter term is far too inclusive.

Emerson, Emerson, and Evans (1936) identified this factor as alphatocopherol, and later Karrer and Demole (1938) synthesized it. Evans and Burr (1928) observed that the offspring of female rats partially deprived of vitamin $E$ often became paralysed toward the end of lactation. It remained for Olcott (1938) to show that the symptoms were due to widespread necrosis and dystrophy in the voluntary muscles. Although some terminal neurites may be destroyed in advanced cases, as shown by Telford (1941), the process affects the muscles primarily. A single dose of $0.5 \mathrm{mg}$. of natural or synthetic tocopherol provides complete protection according to a number of workers cited by Pappenheimer (1943).

Einarson and Ringsted (1938) made an early and extensive study of this condition. In addition to the muscular changes, they described degeneration of the posterior columns and anterior horn cells of the spinal cord, with resultant neural muscular atrophy. More recently, Luttrell and Mason (1949) and Malamud, Nelson, and Evans (1949) have confirmed the eventual occurrence of neural lesions in vitamin E-deficient rats. Other workers, notably Pappenheimer (1943), have been impressed with the primary muscular involvement in the disorder. Different animals show varying kinds and degrees of pathology but most seem to be susceptible, including rats, mice, rabbits, guineapigs, ducklings, and dogs.

Pappenheimer has summarized the pathological changes to be found in the affected muscles. The lesions are widespread but not necessarily symmetrical, and they may not affect an individual muscle in its entirety. In the acute form there is extreme hyaline necrosis with swelling and loss of cross striations. Oedema and a violent cellular reaction may occur. Sarcolemmal nuclei multiply by mitosis and form new myofibrils with every evidence of regeneration. In chronic cases there is a gradual replacement of muscle fibres with fat and fibrous tissue, giving a picture indistinguishable from that of human pseudohypertrophic muscular dystrophy.

From the biochemical viewpoint creatinuria is an outstanding feature, and it is diminished by the administration of tocopherols (Mackenzie and McCollum, 1940). It has also been found that both in vivo and in vitro dystrophic muscle has an 
abnormally high oxygen consumption, and this is corrected by the addition of alpha-tocopherol (Victor, 1934 ; Madsen, 1936 ; Pappenheimer, 1943). Interestingly enough, Brinkhous and Warner (1941) have also shown that dystrophy can be produced in dogs by making a biliary fistula. Here the animals received a normal diet, but diversion of bile apparently interfered with absorption of the fat-soluble vitamin $\mathrm{E}$.

All these findings naturally suggested the trial of vitamin $E$ in a number of human neuromuscular disorders of unknown origin. A flurry of contradictory papers soon appeared. Typical of the early, optimistic reports were those of Wechsler (1940), Bicknell (1940), and Stone (1940). Studies published later, such as those of Doyle and Merritt (1941) and Alpers, Gaskill, and Cantarow (1941) failed to support the early claims.

\section{Materials and Methods}

We report here a long term study of the effect of wheat germ oil in the treatment of 107 patients. The majority suffered from neuromuscular disorders. A breakdown of this material will be found in Table I. Diagnosis was made primarily on careful clinical examination, but special techniques were used in most cases. These included tests of muscle strength and fatiguability, estimations of creatinuria during a meat-free dietary regime, electromyograms, and muscle biopsy examinations. Details of these methods have been described elsewhere (McEachern and Rabinovitch, 1950).

The wheat germ oil used for all cases was supplied by Viobin (Canada) Limited. Their assays claim 2.5 to $3.0 \mathrm{mg}$. of mixed tocopherols per $\mathrm{g}$. $(1.1 \mathrm{ml}$.). The usual dosage was dr. 2 t.i.d. for adults and dr. 1 t.i.d. for children. Since $1 \mathrm{dr}$. is about $3.9 \mathrm{~g}$. of wheat germ oil the usual daily dose of tocopherols was $65 \mathrm{mg}$. for adults and $33 \mathrm{mg}$. for children. This was supplemented in some cases by alphatocopherol acetate ('ephynal' from Roche) and by wheat germ oil concentrated for tocopherols (' phytopherol' from British Drug Houses ; ' germol' $E$ from Ayerst, McKenna, and Harrison). Wheat germ oil concentrate $10: 1$ (Viobin Corporation, Monticello, Illinois), prepared after the method of Milhorat and Bartels (1945), was used as a supplement in some cases. The tocopherol content of this concentrate, as described by the processor, is said to be slightly higher than that of the original wheat germ oil, viz. 3 to $3.5 \mathrm{mg}$. per g. Just what factors are enhanced by the $10: 1$ concentration is not clear. In any event, supplements of some of the above raised the daily intake of tocopherols to $300 \mathrm{mg}$. per day in a few cases. No correlation of effective therapeutic results and tocopherol intake could be noted on this basis, and the results have to be considered in terms of the basic addition of wheat germ oil to the dietary.

Until more is known about the factors in wheat germ oil effective against human disease no claims should be made for individual elements of it without good proof. Even vitamin E itself is a complex containing several tocopherols. If the lesson of the vitamin B complex family is kept in mind this point should be clear.

\section{Results}

Cases Unresponsive to Therapy.-These number 93 out of 107 cases and are listed in Table I. They include all cases of typical progressive motor neuron disease, of myasthenia gravis, of disseminated sclerosis, and a number of other conditions. No favourable response was found in these cases.

Cases Responsive to Therapy.-These include cases of atypical muscular atrophy, progressive muscular dystrophy, menopausal muscular dystrophy, and dermatomyositis.

Atypical Muscular Atrophy.-In the two patients who did show improvement several factors seem to be pertinent. They suggest that a condition other than progressive muscular atrophy prevailed, despite the localized weakness and wasting suggestive of this syndrome.

In case No. 1 bilateral wasting of the forearm and hand muscles appeared rapidly at the age of 20 and remained stationary for 20 years. In the past two years progressive weakness, without wasting, has developed in the lower extremities, coinciding with the onset of the menopause. It may be that the signs in the upper extremities were residues of poliomyelitis, and that the present troubles in the lower extremities are due to superadded menopausal muscular dystrophy.

Case No. 2 presents similar features. Poliomyelitis could account for the long-standing and localized weakness and atrophy. A recent improvement of muscle power in the lower limbs, together with the reduction of fasciculations, is against the diagnosis of progressive muscular atrophy.

CASE No. 1 (M.N.I. 48-5852, D. D., woman, aged 40). -The patient complained of weakness in the lower limbs, slowly progressive for two years. There was pre-existing marked weakness of the upper extremities with a claw hand deformity which had developed fairly rapidly at the age of 20 . Menses recently had been irregular and scanty.

On examination, weakness and wasting, marked in the hand and forearm muscles, moderate in the arm and shoulder muscles bilaterally, was apparent, and also weakness in dorsiflexion of the foot and flexion of the legs. Deep tendon reflexes were absent. Biopsy of the right brachio-radialis showed atrophy of fibres, an increase in the number. of sarcolemmal nuclei, connective tissue replacement, and thickening of blood vessels. 
TABLE I

PATIENTS INVESTIGATED IN THE NEUROMUSCULAR DISEASE CLINIC

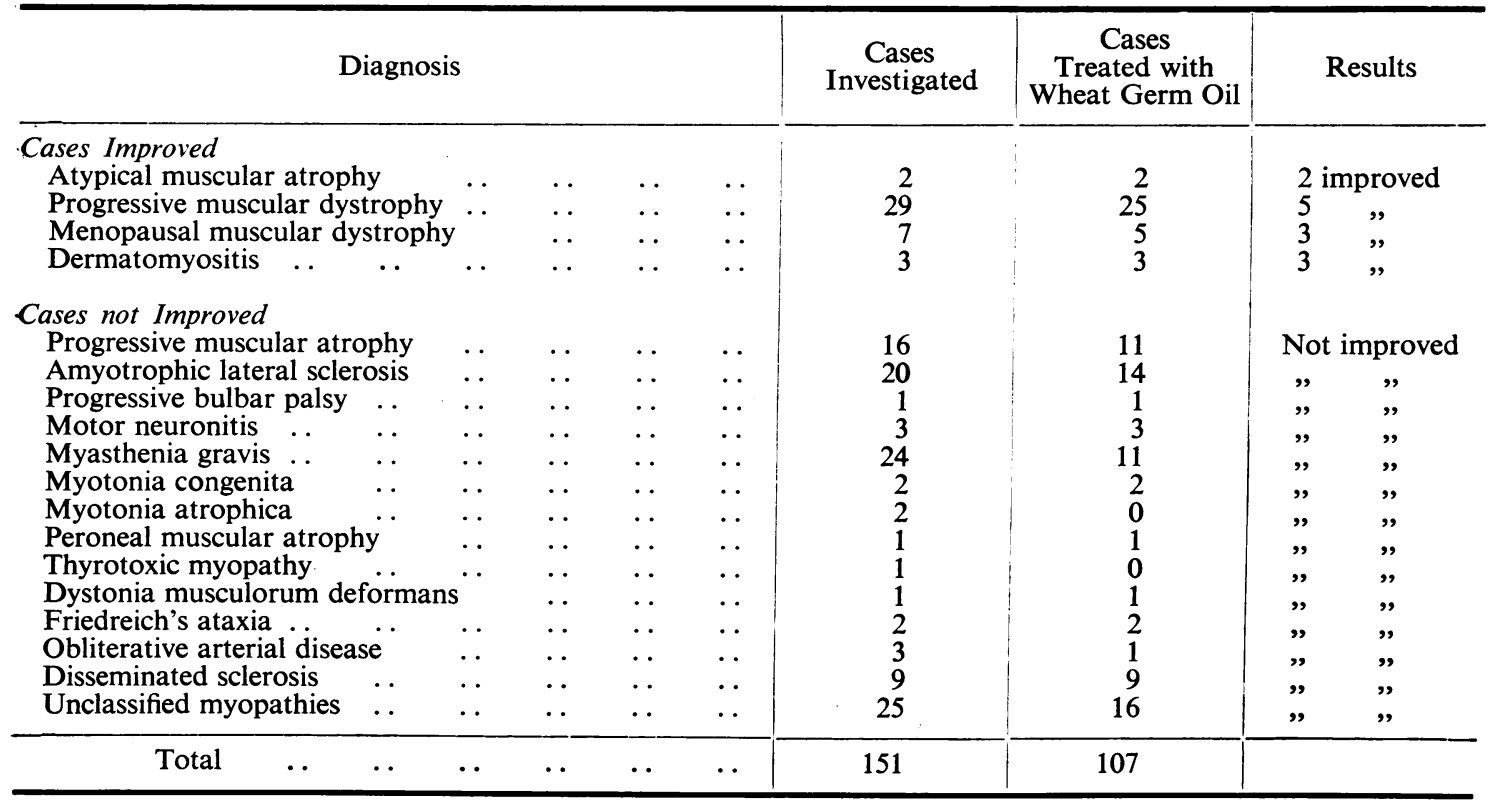

Biopsy of the left gastrocnemius showed variation in size and shape of the fibres, with some loss of cross striation and areas of increased nuclei. An electromyogram showed fairly normal potentials from the right arm and hand muscles, without fibrillation but with large areas of silence. In the left tibialis anticus there were complex motor units and areas of denervation fibrillation. In the right tibialis anticus extremely high voltage motor units and disintegrated motor units were observed.

Wheat germ oil concentrate, dr. 2 t.i.d., was prescribed. After two months there was marked improvement of the leg weakness, and this improvement has persisted for six months. There is no change in the weakness or atrophy of the upper extremities.

CASE No. 2 (M.N.I. 41-7196, s. L., woman, aged 50). - The patient had had poliomyelitis in childhood with weakness and atrophy of the right upper and left lower extremities. She had suffered from generalized fasciculations for eight years, and generalized weakness, nervousness, numbness and tingling in the hands and fingers for one year. Menses had been irregular and scanty for one year.

Examination showed weakness and atrophy of the right shoulder and arm, of both hands and of the left leg muscles. Reflexes were diminished in the right upper and left lower extremities, and there were fasciculations in both lower extremities. Blood pressure was $200 / 120$. Fasciculations were active in the leg muscles. An E.M.G. showed no denervation fibrillation.

Wheat germ oil, dr. 1 t.i.d., was given. Within three months there was improvement of all complaints and this persisted for 18 months. Fasciculations were only faintly visible. Wheat germ oil was discontinued for several months but was begun again when leg weakness reappeared. Improvement was maintained for four years of continuous dosage, but relapse then set in despite the continuance of therapy.

Progressive Muscular Dystrophy.-Five of the 25 patients treated showed sufficient improvement to warrant continuation of treatment with wheat germ oil. In these five patients symptoms appeared in three at about walking age, and in the two others at about the age of 14 . In all five there was involvement of the pelvic girdle muscles, and in one the pectoral girdle was also involved. Although no family history could be elicited, the clinical and laboratory findings agreed with the classical descriptions of progressive muscular dystrophy. Evidence of arrest of the disability with moderate improvement in locomotion seemed quite clear.

CASE No. 1 (O.P.D. 282979, R. T., boy, aged 22 months). -The patient's mother told of his inability to rise from the sitting position. Birth had been normal and also development up to the age of 14 months, at which time he began walking. The mother observed that the child " climbed up his legs" to assume the erect position.

On examination the patient appeared to be a normal child in every respect save for the clumsy manner of rising to his feet. There was "duck-waddle" gait, rubbery consistency of the calf muscles, and absence of all tendon reflexes. 
Wheat germ oil concentrate, dr. 1 q.d., for two months was given, then changed to wheat germ oil, dr. 3 q.d. There was slow improvement, first noticeable two months after treatment began. Eighteen months later he could rise rapidly from the floor, still using his hands. He could walk and run unaided. The "waddle" was less pronounced, and he climbed stairs. The calf muscles were not so rubbery but reflexes were still absent.

CASE No. 2 (o.P.D. G-587, T. K., boy, aged 7).-The child had an abnormal gait since starting to walk, and difficulty in climbing stairs or getting up from a chair.

On examination the patient seemed a well-developed boy who walked with a wide base and a waddling gait. He had difficulty in standing without pushing on his thighs. The calf muscles were of a moderately rubbery consistency.

Wheat germ oil concentrate, dr. $1 \frac{1}{2}$ q.d., was ordered. Improvement in ability to climb stairs and to rise from the floor was noticeable within two months. The improvement persists.

CASE No. 3 (o.P.D. G-6856, A. o'C., boy, aged 7). -The child had a clumsy walk, and difficulty in climbing stairs since the age of $4 \frac{1}{2}$.

On examination it was seen that his walking was fair but he ran on tiptoe. Rising from the floor and climbing stairs was very difficult. There was no atrophy, hypertrophy, or fasciculation of muscles. An electromyogram was normal.

Wheat germ oil concentrate, dr. 3 q.d., was ordered. Improvement was seen within two months and has persisted for seven months. Rising from the floor, walking, running, and climbing stairs were performed more smoothly, and the child experienced less fatigue on effort. He rides a bicycle without difficulty.

CASE No. 4 (O.P.D. G-3434, E. M., man, aged 18).-The patient complained of progressive wasting of the arms and thighs bilaterally for four years following severe scarlet fever. Weakness was first apparent in the lower limbs with frequent falling. He had had articular pain since scarlet fever.

On examination marked weakness bilaterally of the deltoids, biceps, triceps, rhomboids, sacrospinalis, and quadriceps muscles was noted. Wasting was evident in both pectoral and pelvic girdle muscle groups, most marked in the former. All the deep tendon reflexes were absent. There was no hereditary factor. From a muscle biopsy of the right rectus femoris the muscle appeared soft, flabby, avascular, and microscopically the muscle fibres were seen to be unequal in diameter. Cross striations were normal but the number of sarcolemmal nuclei was increased. The capillary network was poorly developed. Many of the medium sized vessels showed proliferation of the medial coat and some of adventitial coverings.

Wheat germ oil concentrate, dr. 4 q.d., was given. After six months of treatment there was an improvement in strength. The patient did not fall, and had been able to do gardening all summer. Objective tests showed definite improvement.
CASE No. 5 (O.P.D. G-6417, G. L., man, aged 28).--The patient said that since the age of 13 he had noticed gradual weakness and wasting of the thigh muscles, clumsiness in walking, and difficulty in rising from the floor from the seated position and in climbing stairs.

Examination showed marked atrophy of the thigh muscles with pseudohypertrophy of calf muscles bilaterally. The thigh flexors were weakest. Muscle biopsy of the right rectus femoris showed hard, dense tissue made up of muscle fibres which had undergone hyalin degeneration. There was marked increase in connective tissue and fat, and in the capillary network. The variation in diameter of muscle fibres was marked, and there was evidence of lymphocytic infiltration. An electromyogram recorded many multiple, low voltage short duration units, with large portions of muscle electrically silent, but no evidence of denervation fibrillation.

Wheat germ oil concentrate, dr. 6 q.d., was ordered. Within one month there was improvement in strength, in ability to walk, and to climb stairs. Treatment has been continued for eight months and improvement continues.

Menopausal Muscular Dystrophy.-These patients are described in the following paper, also several other patients with the same condition who responded to cortisone.

Dermatomyositis.—Lépine (1901) first conceived the idea that dermatomyositis may be primarily a disturbance of the blood vessels. Since then opinions have varied as to the nature of the cutaneous and muscular changes observed. The skin is often glossy, seems to be slightly adherent to the underlying structures, and there may be scaling and evidence of atrophy. Muscular changes may be profound.

Banks (1941) sought a common denominator in scleroderma, disseminated lupus erythematosis, the Libman-Sacks syndrome, and polyarteritis nodosa. This view is receiving attention now because of the effect of cortisone upon these conditions. Clinical improvement and marked reduction of creatinuria was obtained by Milhorat, Weber, and Toscani (1940) in two cases treated with $125 \mathrm{~g}$. of whole wheat germ daily. In a later report by Milhorat, Toscani, and Bartels (1945) creatinuria in three patients with dermatomyositis was reduced by the administration of large amounts of fresh wheat germ. It is important to note that rigorous control of the samples of wheat germ obtained from different sources showed considerable variation in their effects. These authors believe that the effects of wheat germ oil may be dependent upon its method of preparation.

Muscle biopsy findings in dermatomyositis have been reported by Silverman and Powell (1945). They observed an increase in sarcolemmal nuclei, 
infiltration of the interstitial stroma with plasma cells and with small round cells, occasional neutrophils, and phagocytes. Sometimes there was infiltration of the walls of the medium sized vessels.

Three cases of dermatomyositis with generalized muscular weakness and a rubber-like consistency of muscles have been treated with alphatocopherol and wheat germ oil in this clinic. Marked improvement in symptoms and signs was obtained in all instances. In all three patients hyperthyroidism had existed, and in two of them thyroidectomy had been performed before the institution of therapy.

CASE No. 1 (M.N.I. 5700, L. R., man, aged 62).-The patient complained of weakness of the legs, pain in the knees, and cramps in the feet and legs for four years.

Examination showed that the muscles of the forearms, thighs, and calves were firm to touch with skin adherent to the deep fascia. The small joints of the fingers were stiff. A subtotal thyroidectomy had been performed one year previously.

A total of $9.18 \mathrm{~g}$. of alphatocopherol in 90 days was given by mouth and parenterally. After this the muscles were found to be less rubbery, stiffness was diminished, cramps less frequent, and sufficient strength had returned to enable the patient to return to work as a painter.

CASE No. 2 (R.V.H. 41-4450, I.MCG., woman, aged 49). -The patient complained of generalized joint pains, tenderness, and limitation of movement of the right shoulder two months after thyroidectomy.

Examination showed generalized weakness and wasting of muscles, especially in the upper limbs, and a rubberlike induration of the subcutaneous tissues, muscles, and skin. The small joints of the right hand and left shoulder showed some fixation. A radiograph showed no bony changes. A neurological examination gave normal results. B.M.R. was plus $27 \%$. There was some exophthalmos, and a few residual signs of hyperthyroidism persisted.

Wheat germ oil, dr. 3 q.d., was given. Improvement in symptoms was noted within two months. Eighteen months later it was found that the rubbery induration of subcutaneous tissues and muscles had disappeared completely. There was no swelling or limitation of joint movement, or obvious wasting or weakness of muscles.

CASE No. 3 (M.N.I. 42-5504, M. F., woman, aged 47). -The patient complained of neck pain for three weeks, followed by weakness of the muscles of the neck and of the upper extremities for one year.

Examination showed marked thoracic kyphosis, marked atrophy of the pectoralis and trapezius groups and intrinsic muscles of the hands. The patient could not extend the head or hands. Deep tendon reflexes were absent in upper extremities. The skin was remarkably glossy, firm, inelastic, and adherent to the underlying structures.

Wheat germ oil, dr. 4 q.d., was given. There ' was definite improvement in the strength of the neck and arm muscles within two months. The skin became softer, less glossy, and less adherent to the underlying tissues. Improvement persisted for six years, after which she was again admitted to hospital with a diagnosis of chronic thyrotoxicosis with generalized osteoporosis, muscular atrophy, and scleroderma.

\section{Discussion}

It is not our desire to add to the confusion on the effects of wheat germ and vitamin E. Nevertheless, our experience indicates that certain neuromuscular dişorders respond to wheat germ oil in a rather dramatic way. We have not used alphatocopherol alone, but it is possible that similar results might have been obtained with that substance. Nutritional muscular dystrophy in animals is prevented by crystalline alphatocopherol. In several of our cases relapse occurred upon withdrawing wheat germ oil, and improvement set in again when medication was resumed.

The conditions which respond most vividly might be grouped under the general term of collagen diseases, viz. dermatomyositis and menopausal muscular dystrophy. These are probably metabolic in nature rather than inflammatory, and their response to adrenal corticoids, as shown in the next paper, should provide a lead. It may be found that wheat germ factors and tocopherols are building blocks for steroid hormones, or that they contribute to their tissue effects.

One can only speculate upon the mechanism of progressive muscular dystrophy. It may be a metabolic disorder in which some congenital or acquired lack of essential enzyme systems leads to faulty nutrition of muscle. This could explain why overloading with a necessary food factor, e.g. wheat germ oil, might bring improvement in some cases. The work of Milhorat and Bartels (1945) suggested that patients with progressive muscular dystrophy might have a fault of digestion of vitamin $\mathrm{E}$, and that the latter has to be changed or conjugated to produce the anti-dystrophic principle. If this is so, then the treatment of patients with alphatocopherol by the parenteral route might be expected to be ineffective too. It has been shown by a number of workers, including Mackenzie and McCollum (1940), that the feeding of tocopherols to animals with nutritional muscular dystrophy is much more effective than parenteral therapy. The latter workers give evidence, however, that this may be due to poor absorption of the oily solution from the tissues. Injection of the water soluble sodium phosphate of alphatocopherol was tried in two instances and produced prompt improvement. The author concluded that the activity of vitamin $E$ in rabbit dystrophy is largely dependent upon its physical state. 
It is evident from animal experiments that vitamin $E$ is essential for the integrity of skeletal muscle. It is not so clear, however, what part this factor, or lack of it, plays in the biochemical processes of muscle. The most recent information on this point will be found in the symposium on vitamin $E$ held before the New York Academy of Sciences (Ed. Mason, 1949). The recognition of even small groups of human cases responding to wheat germ factors or vitamin $E$ will be of value in establishing the clinical picture of the deficiency in man.

\section{Summary}

One hundred and fifty-one cases of neuromuscular disorder have been investigated and followed for periods varying from many months to 12 years.

One hundred and seven of these patients were treated with either wheat germ oil or wheat germ oil concentrate, and sometimes with the addition of tocopherols. Thirteen showed definite improvement.

Improvement was noted in two cases of atypical muscular atrophy.

In five (three children and two adults) out of 25 patients with progressive muscular dystrophy, symptoms were arrested and moderate to marked improvement occurred.

Three out of five patients with menopausal muscular dystrophy showed remarkable improvement.

All three cases of dermatomyositis responded favourably to wheat germ oil therapy.

The conclusion is that certain neuromuscular disorders respond to wheat germ oil therapy. These conditions are ones which are also apt to show improvement with the administration of adrenal corticoids.

\section{GIBSON, AND DONALD McEACHERN}

\section{REFERENCES}

Alpers, B. J., Gaskill, H. S., and Cantarow, A. (1941). Arch. Neurol. Psychiat., Chicago, 45, 364.

Banks, B. M. (1941). New Engl. J. Med., 225, 433.

Bicknell, F. (1940). Lancet, 1, 10.

Brinkhous, K. M., and Warner, E. D. (1941). Amer. J. Path., 17, 81.

Doyle, A. M., and Merritt, H. H. (1941). Arch. Neurol. Psychiat., Chicago, 45, 672.

Einarson, L., and Ringsted, A. (1938). "Effect of Chronic Vitamin E Deficiency on the Nervous System and the Skeletal Musculature in Adult Rats." Copenhagen and London.

Emerson, O. H., Emerson, G. A., and Evans, H. M. (1936). Science, 83, 421.

Evans, H. M., and Bishop, K. S. (1922). J. metab. Res., 1, 319.

-, and Burr, G. O. (1928). J. biol. Chem., 76, 273. Karrer, P., and Demole, V. (1938). Schweiz. med. Wschr., 68, 954.

Lépine, R. (1901). Rev. Méd., Paris, 21, 426.

Luttrell, C. N., and Mason, K. E. (1949). Ann. N.Y. Acad. Sci., 52, 113.

Mackenzie, C. G., and McCollum, E. V. (1940). J. Nutrit., 19, 345.

Madsen, L. L. (1936). Ibid., 11, 471.

Malamud, N., Nelson, M. M., and Evans, H. M. (1949). Ann. N.Y. Acad. Sci., 52, 135.

Mason, K. E., Editor (1949). "Vitamin E." Ibid., 52, Art. 3, 63-428.

McEachern, D., and Rabinovitch, R. (1950). Advanc. intern. Med., 4, 201.

Milhorat, A. T. (1940). Med. Clin. N. Amer., 24, 769. -, and Bartels, W. E. (1945). Science, 101, 93. , Weber, F. C., and Toscani, V. (1940). Proc. Soc. Exper. Biol., N.Y., 43, 470.

$\longrightarrow$, Toscani, V., and Bartels, W. E. (1945). Ibid., $58,40$.

Olcott, H. S. (1938). J. Nutrit., 15, 221.

Pappenheimer, A. M. (1943). Res. Publ. Ass. nerv. ment. Dis., 22, 85.

Silverman, J. J., and Powell, V. E. (1945). Amer. Heart J., 30, 441 .

Stone, S. (1940). J. Amer. med. Ass., 114, 2187.

Telford, I. R. (1941) Anat. Rec., 81, 171.

Victor, J. (1934). Amer. J. Physiol., 108, 229.

Wechsler, I. S. (1940). J. Amer. med. Ass., 114, 948. 